\title{
Integral BVPs for a Class of First-Order Impulsive Functional Differential Equations
}

\author{
Xiaofei He, ${ }^{1}$ Jingli Xie, ${ }^{1}$ Guoping Chen, ${ }^{1}$ and Jianhua Shen ${ }^{2}$ \\ ${ }^{1}$ Department of Mathematics, College of Zhangiajie, Jishou University, Jishou, Hunan 416000, China \\ 2 Department of Mathematics, Hangzhou Normal University, Hangzhou, Zhejiang 310036, China
}

Correspondence should be addressed to Jianhua Shen, jhshen2ca@yahoo.com

Received 11 November 2009; Accepted 25 January 2010

Academic Editor: Fengde Chen

Copyright (C) 2010 Xiaofei He et al. This is an open access article distributed under the Creative Commons Attribution License, which permits unrestricted use, distribution, and reproduction in any medium, provided the original work is properly cited.

The methods of lower and upper solutions and monotone iterative technique are employed to the study of integral boundary value problems for a class of first-order impulsive functional differential equations. Sufficient conditions are obtained for the existence of extreme solutions.

\section{Introduction and Preliminaries}

In this paper, we study the following integral boundary value problems (BVPs for short) of the impulsive functional differential equation

$$
\begin{gathered}
x^{\prime}(t)+b(t) x(t)=f(t, x(t),[K x](t)), \quad t \neq t_{k}, t \in J=[0, T], \\
\Delta x\left(t_{k}\right)=I_{k}\left(x\left(t_{k}\right)\right), \quad k=1,2, \ldots, m, \\
x(0)+\mu \int_{0}^{T} x(s) d s=x(T), \quad \mu \leq 0,
\end{gathered}
$$

where $f \in C\left(J \times R^{2}, R\right), I_{k} \in C(R, R),(1 \leq k \leq m), b(t) \in C(R), b(t) \leq 0, J=[0, T], 0=$ $t_{0}<t_{1}<t_{2}<\cdots<t_{m}<t_{m+1}=T . K: P C(J) \rightarrow P C(J)$, where $P C(J)=\{u: J \rightarrow R, u$ is continuous for $t \in J, t \neq t_{k}, u\left(t_{i}^{+}\right), u\left(t_{i}^{-}\right)$exist, and $\left.u\left(t_{i}^{-}\right)=u\left(t_{i}\right), i=1,2, \ldots, m\right\}$. Furthermore, we will assume that $K$ is continuous and monotone nondecreasing, and for any bounded set $A \subseteq P C(J), K A$ is bounded. $\Delta x\left(t_{k}\right)=x\left(t_{k}^{+}\right)-x\left(t_{k}^{-}\right)$denotes the jump of $x(t)$ at $t=t_{k}$; $x\left(t_{k}^{+}\right)$and $x\left(t_{k}^{-}\right)$represent the right and left limits of $x(t)$ at $t=t_{k}$, respectively. Denote $J^{\prime}=$ $J \backslash\left\{t_{1}, t_{2}, \ldots, t_{m}\right\}$. 
Let $P C^{1}(J)=\left\{u \in P C(J): u\right.$ be continuously differentiable for $\left.t \in J, t \neq t_{k}\right\} . P C(J)$ and $P C^{1}(J)$ are Banach spaces with the norms

$$
\|u\|_{P C(J)}=\sup \{|u(t)|: t \in J\}, \quad\|u\|_{P C^{1}(J)}=\max \left\{\|u\|_{P C(J)},\left\|u^{\prime}\right\|_{P C(J)}\right\}
$$

By a solution of (1.1) we mean a $u \in P C^{1}(J)$ for which problem (1.1) is satisfied.

Note that (1.1) has a very general form, as special instances resulting from (1.1), one can have impulsive differential equations with deviating arguments and impulsive differential equations with the Volterra or Fredholm operators. When $\mu=0, I_{k} \equiv 0,(1.1)$ reduces to

$$
\begin{gathered}
x^{\prime}(t)+b(t) x(t)=f(t, x(t),[K x](t)), \quad t \in J=[0, T], \\
x(0)=x(T) .
\end{gathered}
$$

In [1], Cao and Li. studied and understood existence and stability of solution of this equation by using fixed theorem and monotone iteration techniques.

When $\mu=0, b(t) \equiv 0,(1.1)$ reduces to

$$
\begin{gathered}
x^{\prime}(t)=f(t, x(t),[K x](t)), \quad t \neq t_{k}, t \in J=[0, T], \\
\Delta x\left(t_{k}\right)=I_{k}\left(x\left(t_{k}\right)\right), \quad k=1,2, \ldots, m, \\
x(0)=x(T) .
\end{gathered}
$$

In [2], Li discussed and built the existence theorem of solutions of this equation by using fixed theorem, upper and lower solutions methods and monotone iterative techniques.

When $\mu=0, b(t) \equiv 0,[K x](t)=x(t)$, the equation (1.1) reduces to the periodic boundary value problem of the impulsive differential equation

$$
\begin{gathered}
x^{\prime}(t)=f(t, x(t)), \quad t \neq t_{k}, \quad t \in J=[0, T], \\
\Delta x\left(t_{k}\right)=I_{k}\left(x\left(t_{k}\right)\right), \quad k=1,2, \ldots, m, \\
x(0)=x(T) .
\end{gathered}
$$

There are plenty of results on studying the periodic boundary value problem of impulsive differential equations (see [3-8]). According to author's know, there are no dependent references for studying the (1.1) yet. To fill in this void, we try to find the conditions on $f$ and $I_{k}$, so that make sure that the (1.1) exists extremal solution.

It is well known that the monotone iterative technique offers an approach for obtaining approximate solutions of nonlinear differential equations, for details, see [4] and the references therein. There also exist several works devoted to the applications of this technique to boundary value problems of impulsive differential equations, see, for example, $[1-3,5-14]$. In this paper, we consider (1.1) by using the method of upper and lower 
solutions combined with monotone iterative technique. This technique plays an important role in constructing monotone sequences which converge to the solutions of our problems. In presence of a lower solution $\alpha$ and an upper solution $\beta$ with $\alpha \leq \beta$, we show under suitable conditions the sequences converge to the solutions of (1.1) by using the method of upper and lower solutions and monotone iterative technique.

Definition 1.1. The functions $\alpha, \beta \in P C^{1}(J)$ are called lower solution and upper solution of (1.1), respectively, if

$$
\begin{gathered}
\alpha^{\prime}(t)+b(t) \alpha(t) \leq f(t, \alpha(t),[K \alpha](t)), \quad t \neq t_{k}, \quad t \in J, \\
\Delta \alpha\left(t_{k}\right) \leq I_{k}\left(\alpha\left(t_{k}\right)\right), \quad k=1,2, \ldots, m, \\
\alpha(0)+\mu \int_{0}^{T} \alpha(s) d s \leq \alpha(T), \quad \mu \leq 0 . \\
\beta^{\prime}(t)+b(t) \beta(t) \geq f(t, \beta(t),[K \beta](t)), \quad t \neq t_{k}, \quad t \in J, \\
\Delta \beta\left(t_{k}\right) \geq I_{k}\left(\beta\left(t_{k}\right)\right), \quad k=1,2, \ldots, m, \\
\beta(0)+\mu \int_{0}^{T} \beta(s) d s \geq \beta(T), \quad \mu \leq 0 .
\end{gathered}
$$

In what follows we define the set

$$
[\alpha, \beta]=\{w \in P C(J, R): \alpha(t) \leq w(t) \leq \beta(t), t \in J\}
$$

for $\alpha, \beta \in P C(J, R)$ and $\alpha \leq \beta$.

We list the following conditions.

$\left(H_{1}\right) \alpha(t), \beta(t)$ are lower and upper solutions of (1.1) such that $\alpha(t) \leq \beta(t)$.

$\left(H_{2}\right)$ There exists $M \geq 0$ such that

$$
f(t, x, y)-f(t, \bar{x}, \bar{y}) \geq-M(x-\bar{x}),
$$

for $\alpha(t) \leq \bar{x} \leq x \leq \beta(t),[T \alpha](t) \leq \bar{y} \leq y \leq[T \beta](t), t \in J$.

$\left(H_{3}\right)$ There exist $0 \leq L_{k}<1, k=1,2, \ldots, m$ such that

$$
I_{k}(x)-I_{k}(y) \geq-L_{k}(x-y), \quad k=1,2, \ldots, m,
$$

for $\alpha(t) \leq y \leq x \leq \beta(t), t \in J$. 


\section{Main Results}

To obtain our main results, we need the following lemmas.

Lemma 2.1 (see [9]). Suppose that the following conditions are satisfied.

$\left(A_{0}\right)$ Sequence $\left\{t_{k}\right\}$ satisfies $0 \leq t_{0}<t_{1}<t_{2} \cdots$, and $\lim _{n \rightarrow \infty} t_{n}=\infty$.

$\left(A_{1}\right) m \in P C^{1}[J, R]$ and $m(t)$ is left continuous at $t_{k}, k=1,2, \ldots$

$\left(A_{2}\right)$ For $k=1,2, \ldots, t \geq t_{0}$,

$$
\begin{gathered}
m^{\prime}(t) \leq p(t) m(t)+q(t), \quad t \neq t_{k}, t \in J, \\
m\left(t_{k}^{+}\right) \leq d_{k} m\left(t_{k}\right)+b_{k}, \quad k=1,2, \ldots, m,
\end{gathered}
$$

where $q, p \in C\left[R_{+}, R\right], b_{k}, d_{k} \geq 0$ are constants, then

$$
\begin{aligned}
m(t) \leq & m\left(t_{0}\right) \prod_{t_{0}<t_{k} \leq t} d_{k} \exp \left(\int_{t_{0}}^{t} p(s) d s\right) \\
& +\sum_{t_{0}<t_{k} \leq t}\left(\prod_{t_{k}<t_{j} \leq t} d_{j} \exp \left(\int_{t_{k}}^{t} p(s) d s\right)\right) b_{k} \\
& +\int_{t_{0}<t_{k}<t}^{t} \prod_{k} \exp \left(\int_{s}^{t} p(\sigma) d \sigma\right) q(s) d s .
\end{aligned}
$$

Lemma 2.2 (see [12]). If $m \in P C^{1}(J)$ and

$$
\begin{gathered}
m^{\prime}(t) \leq-M m(t), \quad t \neq t_{k}, \quad t \in J=[0, T] \\
\Delta m\left(t_{k}\right) \leq-L_{k} m\left(t_{k}\right), \quad k=1,2, \ldots, m \\
m(0) \leq m(T)
\end{gathered}
$$

where $M>0,0<L_{k} \leq 1$, then $m(t) \leq 0, t \in J$.

Lemma 2.3. If $x \in P C(J), M>0,0<L_{k} \leq 1, k=1,2, \ldots, m$, and $\left(1 /\left(1-e^{-M T}\right)\right) \sum_{k=0}^{m} L_{k}<1$, then the equation

$$
\begin{gathered}
x^{\prime}(t)+M x(t)=\sigma(t), \quad t \neq t_{k}, t \in J=[0, T], \\
\Delta x\left(t_{k}\right)=-L_{k} x\left(t_{k}\right)+d_{k}, \quad k=1,2, \ldots, m, \\
x(0)+d=x(T), \quad d \in R
\end{gathered}
$$

has one unique solution. 
Proof. Firstly, we prove that (2.4) is equivalent to the integral equation

$$
x(t)=-\frac{e^{-M t}}{1-e^{-M T}} d+\int_{0}^{T} G(t, s) \sigma(s) d s+\sum_{k=0}^{m} G\left(t, t_{k}\right)\left(-L_{k} x\left(t_{k}\right)+d_{k}\right),
$$

where

$$
G(t, s)= \begin{cases}\frac{e^{-M(t-s)}}{1-e^{-M T},} & 0 \leq s<t \leq T, \\ \frac{e^{-M(T+t-s)}}{1-e^{-M T}}, & 0 \leq t \leq s \leq T .\end{cases}
$$

If $x(t) \in P C^{1}(J)$ is solution of (2.4), then, by directly integrating we obtain

$$
x(t)=-\frac{e^{-M t}}{1-e^{-M T}} d+\int_{0}^{T} G(t, s) \sigma(s) d s+\sum_{k=0}^{m} G\left(t, t_{k}\right)\left(-L_{k} x\left(t_{k}\right)+d_{k}\right) .
$$

If $x(t) \in P C^{1}(J)$ is solution of the above-mentioned integral equation, then

$$
\begin{aligned}
x^{\prime}(t) & =-M\left[-\frac{e^{-M t}}{1-e^{-M T}} d+\int_{0}^{T} G(t, s) \sigma(s) d s+\sum_{k=0}^{m} G\left(t, t_{k}\right)\left(-L_{k} x\left(t_{k}\right)+d_{k}\right)\right]+\sigma(t) \\
& =-M x(t)+\sigma(t), \quad t \neq t_{k}, \\
\Delta x\left(t_{k}\right) & =-L_{k}\left(x\left(t_{k}\right)\right)+d_{k}, \quad k=1,2, \ldots, m, \\
x(0) & =-\frac{1}{1-e^{-M T}} d+\int_{0}^{T} \frac{e^{-M(T-s)}}{1-e^{-M T}} \sigma(s) d s+\sum_{k=0}^{m} \frac{e^{-M\left(T-t_{k}\right)}}{1-e^{-M T}}\left(-L_{k} x\left(t_{k}\right)+d_{k}\right), \\
x(T) & =-\frac{e^{-M T}}{1-e^{-M T}} d+\int_{0}^{T} \frac{e^{-M(T-s)}}{1-e^{-M T}} \sigma(s) d s+\sum_{k=0}^{m} \frac{e^{-M\left(T-t_{k}\right)}}{1-e^{-M T}}\left(-L_{k} x\left(t_{k}\right)+d_{k}\right) .
\end{aligned}
$$

This yields $x(0)+d=x(T)$. So (2.4) is equivalent to the integral equation

$$
x(t)=-\frac{e^{-M t}}{1-e^{-M T}} d+\int_{0}^{T} G(t, s) \sigma(s) d s+\sum_{k=0}^{m} G\left(t, t_{k}\right)\left(-L_{k} x\left(t_{k}\right)+d_{k}\right) .
$$

Now, we define operator $A: P C(J) \rightarrow P C(J)$ as

$$
(A x)(t)=-\frac{e^{-M t}}{1-e^{-M T}} d+\int_{0}^{T} G(t, s) \sigma(s) d s+\sum_{k=0}^{m} G\left(t, t_{k}\right)\left(-L_{k} x\left(t_{k}\right)+d_{k}\right) .
$$


For each $x, y \in P C(J)$,

$$
|(A x)(t)-(A y)(t)| \leq \frac{1}{1-e^{-M T}} \sum_{k=0}^{m} L_{k}|x-y| \leq \frac{1}{1-e^{-M T}} \sum_{k=0}^{m} L_{k}\left\|x\left(t_{k}\right)-y\left(t_{k}\right)\right\|,
$$

and so

$$
\|(A x)(t)-(A y)(t)\| \leq \frac{1}{1-e^{-M T}} \sum_{k=0}^{m} L_{k}\left\|x\left(t_{k}\right)-y\left(t_{k}\right)\right\| .
$$

This indicates that $A: P C(J) \rightarrow P C(J)$ is a contraction mapping. Then there is one unique $x \in P C(J)$ such that $A x=x$, that is, (2.4) has an unique solution $x(t)$. The proof is complete.

Theorem 2.4. If the conditions $\left(H_{1}\right),\left(H_{2}\right),\left(H_{3}\right)$ are all satisfied, and, in addition, if there exist $M>0,0<L_{k} \leq 1, k=1,2, \ldots, m$, such that $\left(1 /\left(1-e^{-M T}\right)\right) \sum_{k=0}^{m} L_{k}<1$, then the impulsive equation (1.1) has minimal and maximal solutions $\rho(t), r(t) \in P C^{1}(J)$ in $[\alpha, \beta]$, and there are monotone sequences $\left\{\alpha_{n}\right\},\left\{\beta_{n}\right\}$ convergeing uniformly to $\rho(t), r(t)$ in $J$, respectively, where $\alpha_{0}=\alpha, \beta_{0}=\beta$, and $\alpha_{n}(t), \beta_{n}(t)$ are lower and upper solutions of $(1.1)$, respectively.

Proof. For each $\psi \in[\alpha, \beta]$, we consider the equation

$$
\begin{gathered}
x^{\prime}(t)=f(t, \psi(t),[K \psi](t))-b(t) \psi(t)-M(x(t)-\psi(t)), \quad t \neq t_{k}, t \in J, \\
\Delta x\left(t_{k}\right)=I_{k}\left(\psi\left(t_{k}\right)\right)-L_{k}\left(x\left(t_{k}\right)-\psi\left(t_{k}\right)\right), \quad k=1,2, \ldots, m, \\
x(0)+\mu \int_{0}^{T} \psi(s) d s=x(T), \quad \mu \leq 0 .
\end{gathered}
$$

By Lemma 2.3, we know that (2.13) has a unique solution $x(t) \in P C^{1}(J)$. Now, we define operator $A: P C^{1}(J) \rightarrow P C^{1}(J)$ as $A \psi=x$.

We will prove that $\left\{\alpha_{n}\right\},\left\{\beta_{n}\right\}$ have the following properties.

(a) $\alpha_{0} \leq A \alpha_{0}, A \beta_{0} \leq \beta_{0}$.

(b) $A$ is monotone nondecreasing on $\left[\alpha_{0}, \beta_{0}\right]$.

Proofs of properties (a), (b) are divided into three steps to proceed.

Step 1. Suppose that $p=\alpha_{0}-\alpha_{1}$, then

$$
\begin{aligned}
p^{\prime}= & \alpha_{0}^{\prime}-\alpha_{1}^{\prime} \leq-b(t) \alpha(t)+f(t, \alpha(t),[K \alpha](t))-f(t, \alpha(t),[K \alpha(t)](t)) \\
& +b(t) \alpha(t)+M\left(\alpha_{1}(t)-\alpha(t)\right)=-M p(t), \quad t \neq t_{k}, \\
\Delta p\left(t_{k}\right)= & \Delta \alpha_{0}-\Delta \alpha_{1} \leq I_{k}\left(\alpha\left(t_{k}\right)\right)-I_{k}\left(\alpha\left(t_{k}\right)\right)+L_{k}\left(\alpha_{1}\left(t_{k}\right)-\alpha\left(t_{k}\right)\right)=-L_{k} p\left(t_{k}\right), \quad t=t_{k}, \\
p(0)= & \alpha_{0}(0)-\alpha_{1}(0) \leq p(T) .
\end{aligned}
$$

By Lemma 2.2, we obtain $p(t) \leq 0, t \in J$, so $\alpha_{0}(t) \leq \alpha_{1}(t)$. 
Step 2. Suppose that $p=\beta_{1}-\beta_{0}$, then

$$
\begin{gathered}
p^{\prime}=\beta_{1}^{\prime}-\beta_{0}^{\prime} \leq b(t) \beta(t)-f(t, \beta(t),[K \beta](t))+f(t, \beta(t),[K \beta(t)](t)) \\
-b(t) \beta(t)-M\left(\beta_{1}(t)-\beta(t)\right)=-M p(t), \quad t=t_{k}, \\
\Delta p\left(t_{k}\right)=\Delta \beta_{1}-\Delta \beta_{0} \leq-I_{k}\left(\beta\left(t_{k}\right)\right)+I_{k}\left(\beta\left(t_{k}\right)\right)-L_{k}\left(\beta_{1}\left(t_{k}\right)-\beta\left(t_{k}\right)\right)=-L_{k} p\left(t_{k}\right), \quad t=t_{k}, \\
p(0)=\beta_{1}(0)-\beta_{0}(0) \leq p(T) .
\end{gathered}
$$

By Lemma 2.2, we obtain $p(t) \leq 0, t \in J$, so $\beta_{1}(t) \leq \beta_{0}(t)$.

Similary we can show that $\alpha_{1}(t) \leq \beta_{1}(t)$, hence $\alpha_{0}(t) \leq \alpha_{1}(t) \leq \beta_{1}(t) \leq \beta_{0}(t)$.

Step 3. If $n=m, \alpha_{m-1} \leq \alpha_{m} \leq \beta_{m} \leq \beta_{m-1}$, then when $n=m+1$, let $p=\alpha_{m}-\alpha_{m+1}$. Then

$$
\begin{aligned}
p^{\prime}= & \alpha_{m}^{\prime}-\alpha_{m+1}^{\prime} \leq-b(t) \alpha_{m-1}(t)+f\left(t, \alpha_{m-1}(t),\left[K \alpha_{m-1}\right](t)\right) \\
& -M\left(\alpha_{m}(t)-\alpha_{m-1}(t)\right)-f\left(t, \alpha_{m}(t),\left[K \alpha_{m}(t)\right](t)\right) \\
& +b(t) \alpha_{m}(t)+M\left(\alpha_{m+1}(t)-\alpha_{m}(t)\right) \\
= & -b(t)\left(\alpha_{m-1}-\alpha_{m}\right)-M p(t), \quad t \neq t_{k} .
\end{aligned}
$$

Furthermore, $b(t) \leq 0, \alpha_{m-1}-\alpha_{m} \leq 0$, thus $p^{\prime}=\alpha_{m}^{\prime}-\alpha_{m+1}^{\prime} \leq-M p(t)$,

$$
\begin{aligned}
\Delta p\left(t_{k}\right)= & \Delta \alpha_{m}-\Delta \alpha_{m+1}=I_{k}\left(\alpha_{m-1}\left(t_{k}\right)\right)-L_{k}\left(\alpha_{m}\left(t_{k}\right)-\alpha_{m-1}\left(t_{k}\right)\right) \\
& -I_{k}\left(\alpha_{m}\left(t_{k}\right)\right)+L_{k}\left(\alpha_{m+1}\left(t_{k}\right)-\alpha_{m}\left(t_{k}\right)\right) \leq-L_{k} p\left(t_{k}\right), \quad t=t_{k}, \\
p(0)= & \alpha_{m}(0)-\alpha_{m+1}(0)=\mu \int_{0}^{T}\left(\alpha_{m}(s)-\alpha_{m-1}(s)\right) d s+p(T) \leq p(T) .
\end{aligned}
$$

By Lemma 2.2, we obtain $p(t) \leq 0, t \in J$, so $\alpha_{m}(t) \leq \alpha_{m+1}(t)$.

Similarly, we can assume that $p=\beta_{m+1}-\beta_{m}$. When $t \neq t_{k}$,

$$
\begin{aligned}
p^{\prime}= & \beta_{m+1}^{\prime}-\beta_{m}^{\prime} \leq-b(t) \beta_{m}(t)+f\left(t, \beta_{m}(t),\left[K \beta_{m}\right](t)\right)-M\left(\beta_{m+1}(t)-\beta_{m}(t)\right) \\
& -f\left(t, \beta_{m-1}(t),\left[K \beta_{m-1}(t)\right](t)\right)+b(t) \beta_{m-1}(t)+M\left(\beta_{m}(t)-\beta_{m-1}(t)\right) \\
= & -b(t)\left(\beta_{m}-\beta_{m-1}\right)-M p(t) .
\end{aligned}
$$

Furthermore, $b(t) \leq 0, \beta_{m}-\beta_{m-1} \leq 0$, thus $p^{\prime}=\beta_{m+1}^{\prime}-\beta_{m}^{\prime} \leq-M p(t)$, when $t=t_{k}$,

$$
\begin{aligned}
\Delta p\left(t_{k}\right)= & \Delta \beta_{m+1}-\Delta \beta_{m}=I_{k}\left(\beta_{m}\left(t_{k}\right)\right)-L_{k}\left(\beta_{m+1}\left(t_{k}\right)-\beta_{m}\left(t_{k}\right)\right) \\
& -I_{k}\left(\beta_{m-1}\left(t_{k}\right)\right)+L_{k}\left(\beta_{m}\left(t_{k}\right)-\beta_{m-1}\left(t_{k}\right)\right) \leq-L_{k} p\left(t_{k}\right), \\
p(0)= & \beta_{m+1}(0)-\beta_{m}(0)=\mu \int_{0}^{T}\left(\beta_{m-1}(s)-\beta_{m}(s)\right) d s+p(T) \leq p(T),
\end{aligned}
$$

hence by Lemma 2.2, we obtain $p(t) \leq 0, t \in J$, so $\beta_{m+1}(t) \leq \beta_{m}(t)$. 
In the same way we can prove that $\alpha_{m+1}(t) \leq \beta_{m+1}(t)$.

Thus by mathematical induction we can know that

$$
\alpha_{n-1} \leq \alpha_{n} \leq \beta_{n} \leq \beta_{n-1}, \quad n=0,1,2, \ldots, t \in J .
$$

So far, we finish the proof of the properties $(a),(b)$.

Now we prove that $\alpha_{n}, \beta_{n}, n=0,1,2, \ldots$, are lower and upper solutions of (1.1). Similarly, we can use mathematical induction to prove this.

When $n=0, \alpha_{0}, \beta_{0}$ are already lower and upper solutions of (1.1).

When $n=1$,

$$
\begin{aligned}
\alpha_{1}(t)= & f(t, \alpha(t),[K \alpha](t))-b(t) \alpha(t)-M\left(\alpha_{1}(t)-\alpha(t)\right) \\
& -f\left(t, \alpha_{1}(t),\left[K \alpha_{1}\right](t)\right)+f\left(t, \alpha_{1}(t),\left[K \alpha_{1}\right](t)\right) \\
\leq & f\left(t, \alpha_{1}(t),\left[K \alpha_{1}\right](t)\right)-b(t) \alpha_{1}(t), \quad t \neq t_{k}, t \in J, \\
\Delta \alpha_{1}\left(t_{k}\right)= & I_{k}\left(\alpha\left(t_{k}\right)\right)-L_{k}\left(\alpha_{1}\left(t_{k}\right)-\alpha\left(t_{k}\right)\right)+I_{k}\left(\alpha_{1}\left(t_{k}\right)\right)-I_{k}\left(\alpha_{1}\left(t_{k}\right)\right) \\
\leq & I_{k}\left(\alpha_{1}\left(t_{k}\right)\right), \quad k=1,2, \ldots, m, \\
& \alpha_{1}(0)+\mu \int_{0}^{T} \alpha_{1}(s) d s \leq \alpha_{1}(0)+\mu \int_{0}^{T} \alpha(s) d s=\alpha_{1}(T), \quad \mu \leq 0 .
\end{aligned}
$$

Thus $\alpha_{1}$ is lower solution of (1.1).

Suppose that $\alpha_{n}$ is lower solution of (1.1) when $n=m$.

Then when $n=m+1$,

$$
\begin{aligned}
\alpha_{m+1}(t)= & f\left(t, \alpha_{m}(t),\left[K \alpha_{m}\right](t)\right)-b(t) \alpha_{m}(t)-M\left(\alpha_{m+1}(t)-\alpha_{m}(t)\right) \\
& -f\left(t, \alpha_{m+1}(t),\left[K \alpha_{m+1}\right](t)\right)+f\left(t, \alpha_{m+1}(t),\left[K \alpha_{m+1}\right](t)\right) \\
\leq & f\left(t, \alpha_{m+1}(t),\left[K \alpha_{m+1}\right](t)\right)-b(t) \alpha_{m+1}(t), \quad t \neq t_{k}, t \in J, \\
\Delta \alpha_{m+1}\left(t_{k}\right)= & I_{k}\left(\alpha_{m}\left(t_{k}\right)\right)-L_{k}\left(\alpha_{m+1}\left(t_{k}\right)-\alpha_{m}\left(t_{k}\right)\right)+I_{k}\left(\alpha_{m+1}\left(t_{k}\right)\right) \\
& -I_{k}\left(\alpha_{m+1}\left(t_{k}\right)\right) \leq I_{k}\left(\alpha_{m+1}\left(t_{k}\right)\right), \quad k=1,2, \ldots, m, \\
& \alpha_{m+1}(0)+\mu \int_{0}^{T} \alpha_{m+1}(s) d s \leq \alpha_{m+1}(0)+\mu \int_{0}^{T} \alpha_{m}(s) d s=\alpha_{m+1}(T) .
\end{aligned}
$$

Thus by mathematical induction we can know that $\alpha_{n}$ is lower solution of (1.1). In the same way we can prove that $\beta_{n}$ is upper solution of (1.1).

By $\alpha_{n-1} \leq \alpha_{n} \leq \beta_{n} \leq \beta_{n-1}, n=0,1,2, \ldots, t \in J$, we can know that when $n \rightarrow+\infty,\left\{\alpha_{n}\right\},\left\{\beta_{n}\right\}$ have limits $\rho(t), r(t)$, respectively. Since they are independent of $t$ when $n \rightarrow+\infty\left\{\alpha_{n}\right\},\left\{\beta_{n}\right\}$ converge uniformly to $\rho(t), r(t)$ and $\alpha_{n} \leq \rho(t) \leq r(t) \leq \beta_{n} \leq \beta_{n-1}, n=$ $0,1,2, \ldots, t \in J$. 
According to $\alpha_{n}, \beta_{n}$ satisfying (2.13), that is,

$$
\begin{gathered}
\alpha_{n}^{\prime}(t)=f\left(t, \alpha_{n-1}(t),\left[K \alpha_{n-1}\right](t)\right)-b(t) \alpha_{n-1}(t)-M\left(\alpha_{n}(t)-\alpha_{n-1}(t)\right), \quad t \neq t_{k}, t \in J, \\
\Delta \alpha_{n}\left(t_{k}\right)=I_{k}\left(\alpha_{n-1}\left(t_{k}\right)\right)-L_{k}\left(\alpha_{n}\left(t_{k}\right)-\alpha_{n-1}\left(t_{k}\right)\right), \quad k=1,2, \ldots, m, \\
\alpha_{n}(0)+\mu \int_{0}^{T} \alpha_{n-1}(s) d s=\alpha_{n}(T), \quad \mu \leq 0, \\
\beta_{n}^{\prime}(t)=f\left(t, \beta_{n-1}(t),\left[K \beta_{n-1}\right](t)\right)-b(t) \beta_{n-1}(t)-M\left(\beta_{n}(t)-\beta_{n-1}(t)\right), \quad t \neq t_{k}, t \in J, \\
\Delta \beta_{n}\left(t_{k}\right)=I_{k}\left(\beta_{n-1}\left(t_{k}\right)\right)-L_{k}\left(\beta_{n}\left(t_{k}\right)-\beta_{n-1}\left(t_{k}\right)\right), \quad k=1,2, \ldots, m, \\
\beta_{n}(0)+\mu \int_{0}^{T} \beta_{n-1}(s) d s=\beta_{n}(T), \quad \mu \leq 0,
\end{gathered}
$$

when $n \rightarrow+\infty$, we have

$$
\begin{aligned}
& \rho^{\prime}(t)=f(t, \rho(t),[K \rho](t))-b(t) \rho(t), \quad t \neq t_{k}, t \in J, \\
& \Delta \rho\left(t_{k}\right)=I_{k}\left(\rho\left(t_{k}\right)\right), \quad k=1,2, \ldots, m, \\
& \rho(0)+\mu \int_{0}^{T} \rho(s) d s=\rho(T), \quad \mu \leq 0 . \\
& r^{\prime}(t)=f(t, r(t),[K r](t))-b(t) r(t), \quad t \neq t_{k}, t \in J, \\
& \Delta r\left(t_{k}\right)=I_{k}\left(r\left(t_{k}\right)\right), \quad k=1,2, \ldots, m, \\
& r(0)+\mu \int_{0}^{T} r(s) d s=r(T), \quad \mu \leq 0 .
\end{aligned}
$$

Equation (2.24) indicates that $\rho(t), r(t)$ are solutions of (1.1). in $[\alpha, \beta]$.

Lastly, we prove that $\rho(t), r(t)$ are minimal and maximal solutions of the equation (1.1)

Suppose that $x(t)$ is a solution of the equation and satisfies $x(t) \in[\alpha, \beta], t \in J$, obviously, we can assume that there is an $n$ such that $\alpha_{n} \leq x \leq \beta_{n}$.

If $p(t)=\alpha_{n+1}-x$, then

$$
\begin{aligned}
p^{\prime}= & \alpha_{n+1}^{\prime}-x^{\prime} \leq-b(t) \alpha_{n}(t)+f\left(t, \alpha_{n}(t),\left[K \alpha_{n}\right](t)\right) \\
& -M\left(\alpha_{n+1}(t)-\alpha_{n}(t)\right)-f(t, x(t),[K x(t)](t))+b(t) x(t) \\
\leq & -b(t)\left(\alpha_{n}-x\right)-M p(t), t \neq t_{k} .
\end{aligned}
$$


And since $b(t) \leq 0, \alpha_{m}-x \leq 0, p^{\prime} \leq-M p(t)$,

$$
\begin{gathered}
\Delta p\left(t_{k}\right)=\Delta \alpha_{n+1}-\Delta x=I_{k}\left(\alpha_{n}\left(t_{k}\right)\right)-L_{k}\left(\alpha_{n+1}\left(t_{k}\right)-\alpha_{n}\left(t_{k}\right)\right)-I_{k}\left(x\left(t_{k}\right)\right) \leq-L_{k} p\left(t_{k}\right), \quad t=t_{k} \\
p(0)=\alpha_{n+1}(0)-x(0)=\mu \int_{0}^{T}\left(x(s)-\alpha_{n}(s)\right) d s+p(T) \leq p(T) .
\end{gathered}
$$

Hence by Lemma 2.2, we can obtain $p(t) \leq 0, t \in J$, so $\alpha_{n+1}(t) \leq x(t)$. Similarly, we can obtain: $x(t) \leq \beta_{n+1}(t), t \in J$. This indicates that $\alpha_{n}(t) \leq x(t) \leq \beta_{n+1}(t), t \in J, n=0,1,2, \ldots$. Hence when $n \rightarrow+\infty$, we can obtain that $\rho(t) \leq x(t) \leq r(t), t \in J$. This ends the proof.

Finally, we give an example to illustrate the efficiency of our results.

Example 2.5. Consider the problem of

$$
\begin{gathered}
x^{\prime}(t)-x(t) \sin t=-x(t)+t+\int_{0}^{t} x(s) d s, \quad 0<t<1, \quad t \neq t_{1}, \\
\Delta x\left(t_{1}\right)=-\frac{1}{8} x\left(t_{1}\right), \quad t_{1}=\frac{1}{2} \\
x(0)-\int_{0}^{1} x(s) d s=x(1)
\end{gathered}
$$

where $b(t)=\sin t, f(t, x(t), K x(t))=-x(t)+t+\int_{0}^{t} x(s) d s, I_{1}(x)=-x$. Obviously, $\alpha(t)=$ $0, \beta(t)=1-t$ are the lower solution and upper solution for (2.27) with $\alpha(t) \leq \beta(t)$, respectively. $f(t, x, K x)-f(t, y, K y)=-(x-y)-\int_{0}^{t}(x(s)-y(s)) d s, I_{1}(x)-I_{1}(y)=-(x-y)$. Let $T=1, L_{k}=1 / 8$, the conditions of Theorem 2.4 are all satisfied, so problem (2.27) has the maximal and minimal solutions in the segement $[\alpha(t), \beta(t)]$.

\section{Acknowledgments}

This work was supported by the NNSF of China (10571050; 10871062), the Hunan Provincial Natural Science Foundation of China (09JJ6010), and the Zhejiang Provincial Natural Science Foundation of China (Y6090057).

\section{References}

[1] S. C. Cao and H. J. Li, "Periodic boundary value problem for first order integro-differential equations," in Proceedings of the 5th International Conference on Impulsive $\mathcal{E}$ Hybrid Dynamical Systems and Applications, pp. 86-89, 2008.

[2] X. Z. Liu, "Nonlinear boundary value problems for first order impulsive integro-differential equations," Journal of Applied Mathematics and Simulation, vol. 2, no. 3, pp. 185-198, 1989.

[3] I. Rachunkova and M. Tvrdy, "Existence results for impulsive second-order periodic problems," Nonlinear Analysis: Theory, Methods \& Applications, vol. 59, no. 1-2, pp. 133-146, 2004.

[4] S. G. Hristova and G. K. Kulev, "Quasilinearization of a boundary value problem for impulsive differential equations," Journal of Computational and Applied Mathematics, vol. 132, no. 2, pp. 399-407, 2001. 
[5] Z. M. He and X. M. He, "Periodic boundary value problems for first order impulsive integrodifferential equations of mixed type," Journal of Mathematical Analysis and Applications, vol. 296, no. 1, pp. 8-20, 2004.

[6] D. Jiang and J. Wei, "Monotone method for first- and second-order periodic boundary value problems and periodic solutions of functional differential equations," Nonlinear Analysis: Theory, Methods $\mathcal{E}$ Applications, vol. 50, pp. 885-898, 2002.

[7] G. P. Chen and J. H. Shen, "Integral boundary value problems for first order impulsive functional differential equations," International Journal of Mathematical Analysis, vol. 1, no. 17-20, pp. 965-974, 2007.

[8] Z. M. He and X. M. He, "Monotone iterative technique for impulsive integro-differential equations with periodic boundary conditions," Computers \& Mathematics with Applications, vol. 48, no. 1-2, pp. 73-84, 2004.

[9] V. Lakshmikantham, D. D. Bainov, and P. S. Simeonov, Theory of Impulsive Differential Equations, vol. 6, World Scientific, Singapore, 1989.

[10] M. P. Yao and F. Q. Zhang, "Integral boundary value problems for a class of first-order impulsive differential equations," Journal of North University of China, vol. 27, no. 2, pp. 138-140, 2006.

[11] D. D. Bainov and P. S. Simeonov, Systems with Impulse Effect: Stability, Theory and Applications, Halsted Press, New York, NY, USA, 1989.

[12] D. D. Bainov and P. S. Simeonov, Impulsive Differential Equations: Periodic Solutions and Applications, Pitman Monographs and Surveys in Pure and Applied Mathematics, Longman Scientific and Technical, Harlow, UK, 1993.

[13] D. D. Bainov and P. S. Simeonov, Impulsive Differential Equations, vol. 28, World Scientific, Singapore, 1995.

[14] X. Z. Liu and A. Willms, "Impulsive controllability of linear dynamical systems with applications to maneuvers of spacecraft," Mathematical Problems in Engineering, vol. 8, pp. 12-32, 1996. 


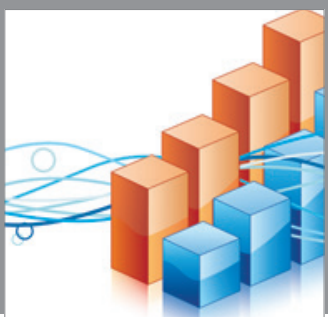

Advances in

Operations Research

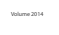

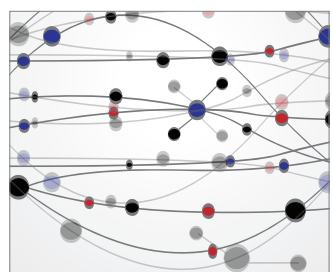

\section{The Scientific} World Journal
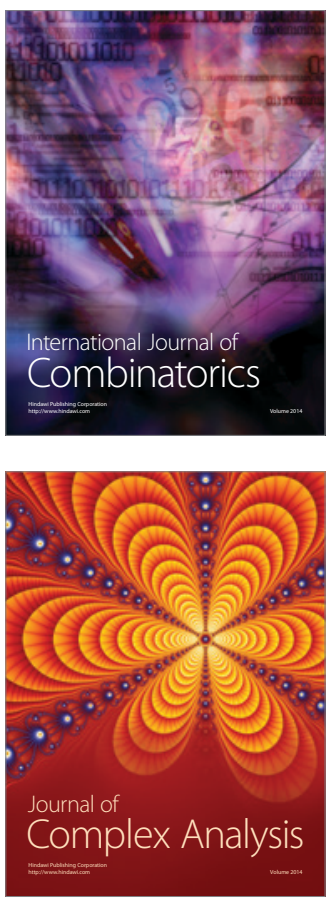

International Journal of

Mathematics and

Mathematical

Sciences
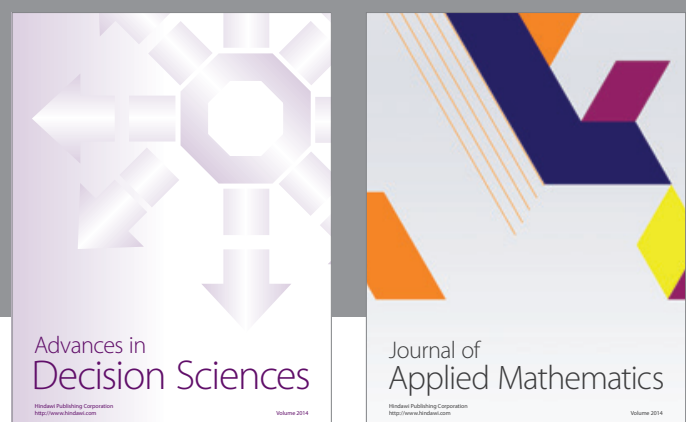

Journal of

Applied Mathematics
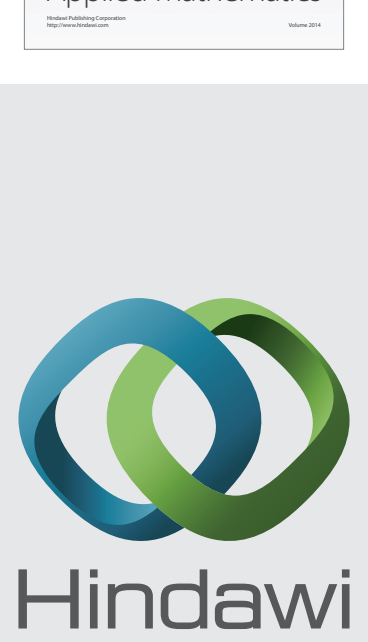

Submit your manuscripts at http://www.hindawi.com
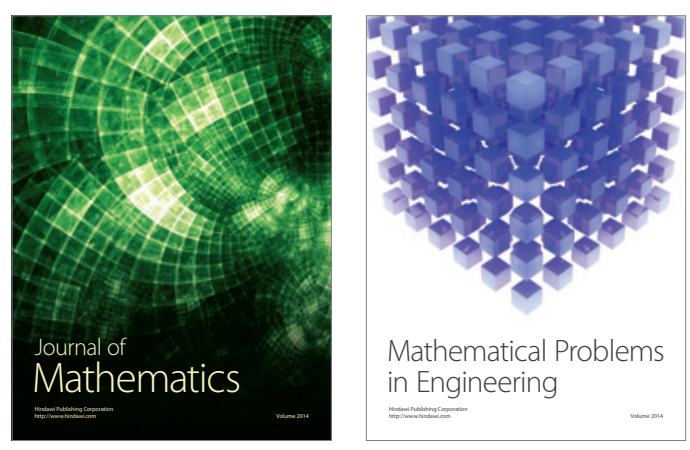

Mathematical Problems in Engineering
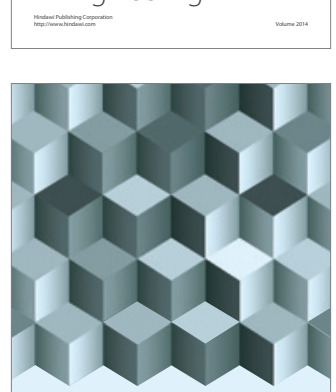

Journal of

Function Spaces
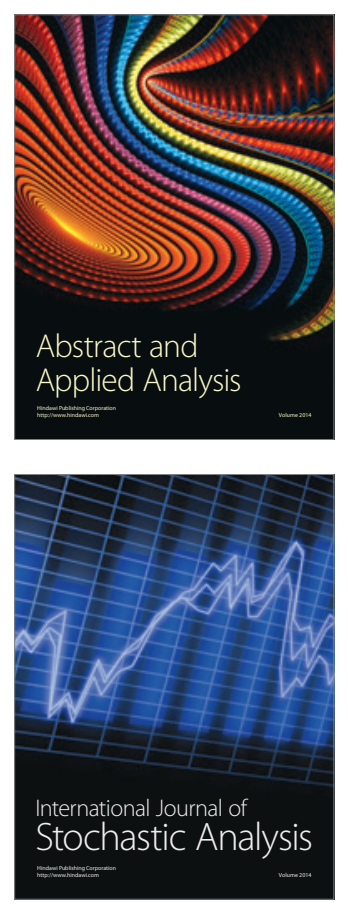

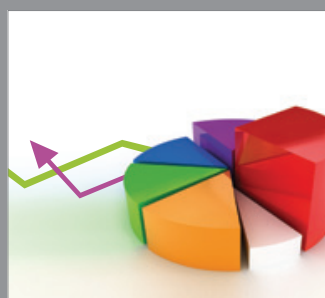

ournal of

Probability and Statistics

Promensencen
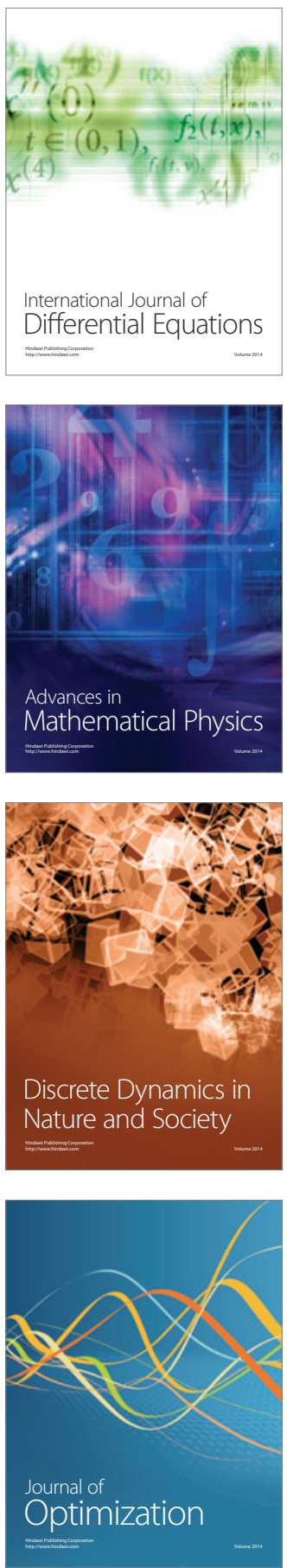